\title{
Signature of rotors
}

\author{
by \\ Mieczysław K. Dąbkowski (Richardson, TX), \\ Makiko Ishiwata (Tokyo), Józef H. Przytycki (Washington, DC) \\ and Akira Yasuhara (Tokyo)
}

\begin{abstract}
Rotors were introduced as a generalization of mutation by Anstee, Przytycki and Rolfsen in 1987. In this paper we show that the Tristram-Levine signature is preserved by orientation-preserving rotations. Moreover, we show that any link invariant obtained from the characteristic polynomial of the Goeritz matrix, including the Murasugi-Trotter signature, is not changed by rotations. In 2001, P. Traczyk showed that the Conway polynomials of any pair of orientation-preserving rotants coincide. We show that there is a pair of orientation-reversing rotants with different Conway polynomials.
\end{abstract}

1. Introduction. Rotors were introduced in graph theory by W. Tutte [2], [17] and [18]. The concept was adapted to knot theory in [1] as a generalization of Conway's mutation. For the orientation of the boundary of an oriented rotor, we have two basic possibilities:

(a) Inputs and outputs alternate as in Fig. 2.2(a). Such a rotor is said to be orientation-preserving.

(b) We have the pattern in-in, out-out as in Fig. 2.2(b). Such a rotor is said to be orientation-reversing $\left(^{1}\right)$.

In Section 3 (resp. 4), we show, in particular, that Murasugi's unoriented version of the classical signature $[4,10,11]$ (Theorem 3.1) (resp. TristramLevine signature) is preserved by any rotations (resp. any orientation-preserving rotations).

It was shown in [1] that rotations of order three and four preserve the Homflypt polynomial, and in particular, the Conway polynomial of links. In 2001, P. Traczyk [14] showed that the Conway polynomials of a pair of

2000 Mathematics Subject Classification: Primary 57M27; Secondary 57M25.

Key words and phrases: link, mutation, rotor, signature, Seifert form, Goeritz form, branched cover, Conway polynomial, Jones polynomial.

Research of M. Ishiwata and A. Yasuhara partially sponsored by the Sumitomo Foundation (030550).

$\left({ }^{1}\right)$ The terminology used here is explained in Section 2. 
any orientation-preserving rotants coincide, solving the Jin-Rolfsen Conjecture [6] in this case. But it was not known if orientation-reversing rotations have the same property for $n \geq 6$. In the last section, we present an example of orientation-reversing rotants which do not share the same Conway polynomial. This disproves the Jin-Rolfsen Conjecture in the orientation-reversing case $[6,12]$.

In general, it is not true that a rotation preserves the first homology of the double branched cover, $M_{L}^{(2)}$, of $S^{3}$ branched along $L$. Necessary conditions for preserving the homology are given in [3, 13]. Figure 1.1 taken from [3] shows rotants with different $H_{1}\left(M_{L_{k}}^{(2)} ; \mathbb{Z}\right)$ and $H_{1}\left(M_{L_{k}}^{(2)} ; \mathbb{Z}_{5}\right)$. For the link $L_{1}$ in Fig. 1.1(a), $H_{1}\left(M_{L_{1}}^{(2)} ; \mathbb{Z}\right)=\mathbb{Z}_{15} \oplus \mathbb{Z}_{30}$ and $H_{1}\left(M_{L_{1}}^{(2)} ; \mathbb{Z}_{5}\right)=\mathbb{Z}_{5} \oplus \mathbb{Z}_{5}$, and for its orientation-preserving rotant $L_{2}$ in Fig. 1.1(b) we obtain $H_{1}\left(M_{L_{2}}^{(2)} ; \mathbb{Z}\right)=$ $\mathbb{Z}_{3} \oplus \mathbb{Z}_{150}$ and $H_{1}\left(M_{L_{2}}^{(2)} ; \mathbb{Z}_{5}\right)=\mathbb{Z}_{5}$. All the homology groups were calculated using K. Kodama's program KNOT [7].

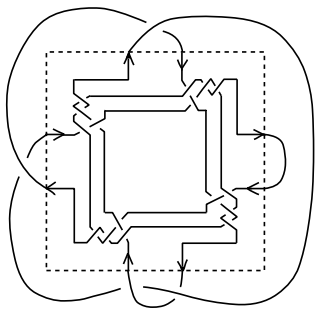

$L_{1}$

(a)

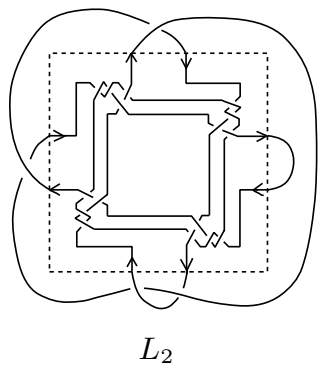

(b)

Fig. 1.1

However, if we assume that a given pair of oriented rotants can be put into a "special" periodic disk-band form then the relevant first homology groups are isomorphic (Corollary 2.3).

2. Definitions and basic properties of rotors. For an oriented link $L$ with $k$ components $K_{1}, \ldots, K_{k}$ we form the linking matrix $A_{L}$ with entries $a_{i j}=\operatorname{lk}\left(K_{i}, K_{j}\right)$, where $i \neq j$. We put $a_{i i}=0$ unless $L$ is a framed link; in that case we define $a_{i i}$ to be the framing of the $i$ th component $K_{i}$ of $L$ ( $a_{i i}$ measures the difference with respect to the standard framing). The linking matrix $A_{L}$, up to the order of the components of $L$, is a link invariant. One half of the sum $\sum_{i<j} a_{i j}$ of the entries of $A_{L}$ off the diagonal is the total linking number of $L$, denoted by $\operatorname{lk}(L)$. The trace of $A_{L}$ for a framed link $L$ is denoted by $\operatorname{tr}(L)$. Note that $\operatorname{tr}(L)$ does not depend on the orientation of $L$, so it is an invariant of the unoriented framed link $L$. 
Consider a link $L$ in $S^{3}$ decomposed into two $n$-tangles $(n>2) S$ and $R$ (Fig. 2.1), where by an $n$-tangle we mean any 1 -dimensional manifold properly embedded into a three-ball and consisting of $n$-arcs and, possibly, closed components. Let $\phi$ be a rotation of $B^{3}=B^{2} \times I$ through $2 \pi / n$ about the $z$ axis. Assume that $R$, called the rotor part of $L$, satisfies $\phi(R)=R$. The other tangle part, $S$, of $L$ is called the stator. Equivalently, $L$ admits a projection decomposed into the projections of the rotor and the stator (these projections will also be denoted by $S$ and $R$ ) such that $R$ lies in a regular $n$-gon and intersects its boundary in $2 n$ points, and $\phi(R)=R$ (Fig. 2.1).

The group of symmetries of the regular $n$-gon is the dihedral group $D_{2 n}$. This group is generated by the $2 \pi / n$ rotation about the $z$ axis $\phi$ and the dihedral flype $d_{0}$ which corresponds to the rotation through $\pi$ about the $y$ axis. The group $D_{2 n}$ has a presentation $D_{2 n}=\left\langle\phi, d_{0}\right| \phi^{n}=d_{0}^{2}=1, d_{0} \phi d_{0}=$ $\left.\phi^{-1}\right\rangle$. Let $d_{k / 2}=\phi^{k} d_{0}$. Note that $d_{k / 2}$ is the dihedral flype about the axis obtained from the $y$ axis by rotating it counterclockwise through the angle $\frac{2 \pi k}{2 n}$.

A rotant of a link $L_{1}$ is the link $L_{2}$ (Figs. 1.1 and 2.1) obtained from $L_{1}$ by a dihedral flype of its rotor part. Note that $L_{2}$ is independent of the choice of a dihedral flype. We say that $L_{2}$ is obtained from $L_{1}$ by a rotation.

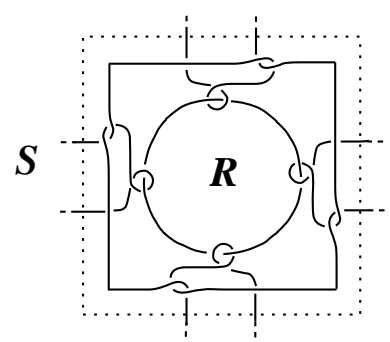

$L_{1}$

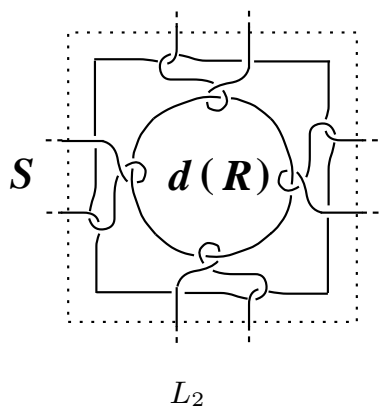

$L_{2}$

Fig. 2.1

If a link is equipped with additional structures such as orientation or a blackboard framing, we also assume that the rotation preserves these structures. In the oriented case, we allow a global change of the orientation of the rotor part. More precisely, for an oriented rotor we have two basic choices of directions of arcs at its boundary points: either inputs and outputs alternate as in Fig. 2.2(a), we call such a rotor orientation-preserving, or we have the pattern in-in, out-out, ..., in-in, out-out for an even $n$ as in Fig. 2.2(b); we call such a rotor orientation-reversing. For an oriented rotor $R$ of an oriented link $L$ and a dihedral flype $d$, the orientations of $d(R)$ and the stator parts do not always match. If they do not match, then by reversing the orientation of 
$d(R)$, we obtain an oriented link $L_{2}=d(R) \cup S$ that we also call the oriented rotant of $L_{1}$.

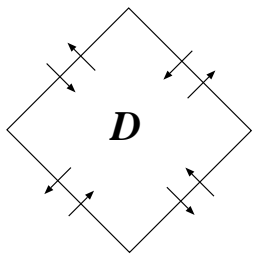

(a)

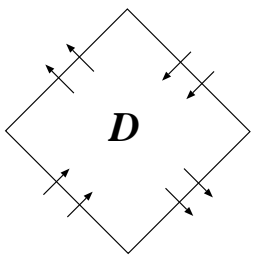

(b)

Fig. 2.2

The following theorem describes the basic properties of rotors.

THEOREM 2.1. (i) Any rotation preserves the number of components of a link.

(ii) If two oriented links are related by a rotation of an oriented rotor, then their total linking numbers are the same.

(iii) If two oriented framed links are related by a rotation of an oriented rotor, and the rotor part has no closed components, then their linking matrices are the same.

(iv) If $L$ is an unoriented framed link, then $\operatorname{tr}(L)$ is preserved by any rotation.

Proof. Let $R$ be an unoriented rotor with boundary points $a_{0}, b_{0}, a_{1}, b_{1}$, $\ldots, a_{n-1}, b_{n-1}$, as in Figure 2.3(a). Consider the connection of $a_{0}$, that is, the boundary point connected to $a_{0}$ by an arc in $R$. Initially, we have two cases: $a_{0}$ connects to either $a_{m}$ or $b_{m}$ for some $m$.

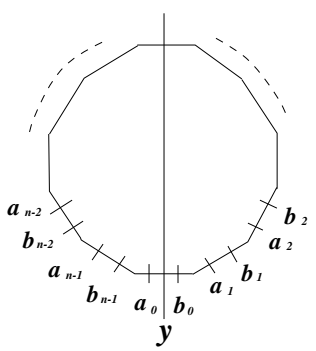

(a)

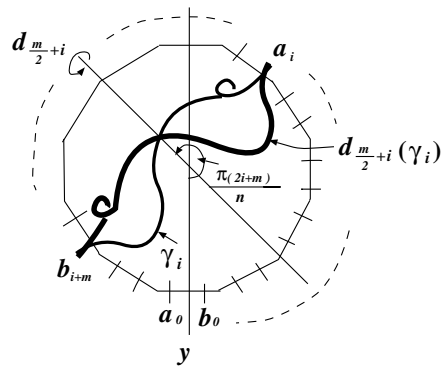

(b)

Fig. 2.3

If $n>2$ then $a_{0}$ cannot be connected to $a_{m}$. To prove this, assume, by contradiction, that $a_{0}$ connects to $a_{m}$; then $\phi^{m}\left(a_{0}\right)=a_{m}$ connects to $\phi^{m}\left(a_{m}\right)=a_{2 m}$, which must be the same as $a_{0}$. Therefore, $2 m=n$. This 
implies that $a_{i}$ connects to $a_{i+n / 2}$ and $b_{i}$ to $b_{i+n / 2}$. The $\operatorname{arc} \gamma\left(x_{i}\right)$ of $R$ connecting $x_{i}$ to $x_{i+n / 2}$, where the symbol $x$ may stand for $a$ or $b$, is setwise preserved by the rotation $\phi^{n / 2}$. Therefore the arc $\gamma\left(x_{i}\right)$ has one fixed point, namely the point of intersection with the $z$ axis. For $n>2$ we have at least two arcs of the type $\gamma\left(a_{i}\right)$. Such arcs meet the $z$ axis at different heights, say $h_{i}$. On the other hand, $\phi\left(\gamma\left(a_{i}\right)\right)=\gamma\left(a_{i+1}\right)$, so $h_{i}=h_{i+1}$, which gives a contradiction. So if $a_{0}$ is connected to $a_{m}$, then $n=2$, and in this case Theorem 2.1 follows easily.

Suppose $a_{0}$ is connected to $b_{m}$ for some $m$. Let $\gamma_{i}=\gamma\left(a_{i}\right)$ denote the arc connecting $a_{i}$ with $b_{i+m}$ in $R$. Consider the dihedral flype $d_{m / 2+i}$ exchanging $a_{i}$ with $b_{i+m}$. The image $d_{m / 2+i}\left(\gamma_{i}\right)$ connects the same points on the boundary as $\gamma_{i}$ does, that is, $a_{i}$ and $b_{i+m}$ (Fig. 2.3(b)), so two boundary points of $R$ are connected in $R$ if and only if they are connected in $d_{0}(R)=d_{m / 2+i}(R)$. In particular, the link $L_{1}=S \cup R$ and its rotant $L_{2}=S \cup d_{0}(R)$ have the same number of components.

By observations similar to the above, we have

Claim 2.2. (i) For an unoriented rotor $R$ choose any orientation (directions) of its arcs (e.g. from $a_{j}$ to $\left.b_{j+m}\right)$. Let $I\left(\gamma_{j}, \gamma_{k}\right)$ denote the sum of the signs of the crossings of $\gamma_{j}$ and $\gamma_{k}$ (possibly $\left.j=k\right)$. Then

$$
I\left(\gamma_{j}, \gamma_{k}\right)=I\left(d_{(j+k+m) / 2}\left(\gamma_{j}\right), d_{(j+k+m) / 2}\left(\gamma_{k}\right)\right) .
$$

(ii) For an oriented rotor $R$ and a closed component $\alpha$ of $R$,

$$
I\left(\gamma_{i}, \alpha\right)=I\left(d_{m / 2+i}\left(\gamma_{i}\right), d_{m / 2+i}(\alpha)\right) .
$$

Notice that $\partial \gamma_{j}=\partial\left(d_{(j+k+m) / 2}\left(\gamma_{k}\right)\right), \partial \gamma_{k}=\partial\left(d_{(j+k+m) / 2}\left(\gamma_{j}\right)\right)$ and $\partial \gamma_{i}=$ $\partial\left(d_{m / 2+i}\left(\gamma_{i}\right)\right)$.

Proof. (i) The dihedral flype $d_{(j+k+m) / 2}$ of $R$ sends $a_{j}$ to $b_{k+m}$ and $a_{k}$ to $b_{j+m}$, thus it sends the arc $\gamma_{j}$, connecting $a_{j}$ with $b_{j+m}$ in $R$ (resp. $a_{k}$ with $\left.b_{k+m}\right)$ to the arc $d_{(j+k+m) / 2}\left(\gamma_{k}\right)$ connecting $b_{k+m}$ with $a_{k}$ in $d_{0}(R)$ (resp. $d_{(j+k+m) / 2}\left(\gamma_{j}\right)$ connecting $b_{j+m}$ with $\left.a_{k}\right)$ (Fig. 2.4). Therefore $I\left(\gamma_{j}, \gamma_{k}\right)=$ $I\left(d_{(j+k+m) / 2}\left(\gamma_{k}\right), d_{(j+k+m) / 2}\left(\gamma_{j}\right)\right)$, as required.

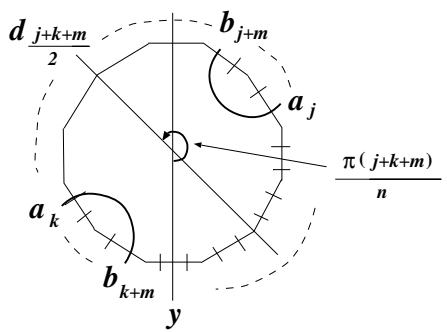

Fig. 2.4 
(ii) Since $\gamma_{i}$ in $R$ and $d_{m / 2+i}\left(\gamma_{i}\right)$ in $d_{0}(R)$ connect the same boundary points $a_{i}$ and $b_{i+m}$, we have the conclusion.

Theorem 2.1(ii)-(iv) follows from Claim 2.2 and the fact that $L_{1}$ and $L_{2}$ have the same stator.

We use Theorem 2.1 to show that under some technical assumptions explained below, the double branched covers of $S^{3}$ branched along rotant links have isomorphic first homology groups. The result of Corollary 2.3 is not used later in the paper; however, we would like to contrast it with the example in Fig. 1.1 of rotant links with different first homology groups.

In the proof of Corollary 2.3 we use Montesinos's method [9] of finding a surgery description of the double branched covers of $S^{3}$ branched along links, when a surface (possibly unoriented) bounding the link is given. We closely follow, in this part of the paper, the notation used in [5].

Let $T_{0}$ be a trivial $n$-tangle diagram as in Fig. 2.5(a). Let $D_{1} \cup \cdots \cup D_{n}$ be a disjoint union of disks bounded by $T_{0}$ and a disjoint union of arcs in $\partial B^{3}$ connecting $\partial T_{0}$. Let $b_{1}, \ldots, b_{m}$ be mutually disjoint disks (ribbons) in $B^{3}$ such that $b_{i} \cap \bigcup_{j} D_{j}=\partial b_{i} \cap T_{0}$ are two disjoint arcs in $\partial b_{i}(i=1, \ldots, m)$ (see Fig. 2.5(b)). We denote by $\Omega\left(T_{0} ;\left\{D_{1}, \ldots, D_{n}\right\},\left\{b_{1}, \ldots, b_{m}\right\}\right)$ the tangle $T_{0} \cup \bigcup_{i} \partial b_{i}-\operatorname{int}\left(T_{0} \cap \bigcup_{i} \partial b_{i}\right)$ together with the the surface $\bigcup D_{i} \cup \bigcup b_{j}$ and its decomposition into disks $D_{i}$ and $b_{i}$. We call such a structure a disk-band representation of a tangle [5].

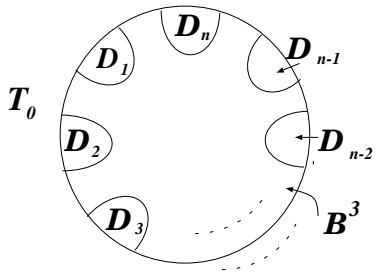

(a)

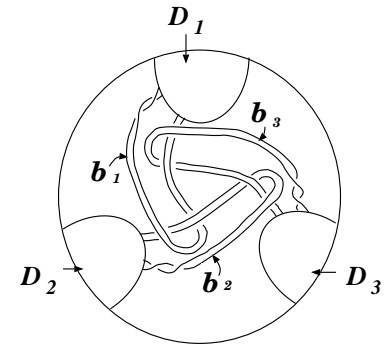

(b)

Fig. 2.5

If a rotor part has a rotationally symmetric disk-band representation, then the following corollary of Theorem 2.1 holds.

Corollary 2.3. Let $L_{1}$ and $L_{2}$ be a pair of unoriented n-rotants such that the $n$-rotor $R_{1}$ of $L_{1}$ admits a rotational symmetric disk-band representation with $n$ ribbon disks. Then $H_{1}\left(M_{L_{1}}^{(2)}, \mathbb{Z}\right)=H_{1}\left(M_{L_{2}}^{(2)} ; \mathbb{Z}\right)$, where $M_{L}^{(2)}$ denotes the double branched cover of $S^{3}$ branched along a link $L$. 
Proof. Let $\Omega\left(T_{0} ;\left\{D_{1}, \ldots, D_{n}\right\},\left\{b_{k 1}, \ldots, b_{k n}\right\}\right)(k=1,2)$ be the the diskband representations of $R_{1}$ and $R_{2}=d_{0}\left(R_{1}\right)$ respectively, related by the dihedral flype $d_{0}$. Let $B^{3}$ be the 3-ball such that $B^{3} \cap L_{k}$ is the tangle ingredient of $\Omega\left(T_{0} ;\left\{D_{1}, \ldots, D_{n}\right\},\left\{b_{k 1}, \ldots, b_{k n}\right\}\right)(k=1,2)$ and $B_{0}^{3}=B^{3}-\operatorname{int} N\left(D_{1} \cup\right.$ $\left.\cdots \cup D_{n}\right)$, where $N\left(D_{1} \cup \cdots \cup D_{n}\right)$ is a regular neighborhood of $D_{1} \cup \cdots \cup D_{n}$ in $B^{3}$. There are compact, connected, possibly nonorientable surfaces $F_{k}$ $(k=1,2)$ in $S^{3}$ such that $F_{k} \cap B^{3}=D_{1} \cup \cdots \cup D_{n} \cup b_{k 1} \cup \cdots \cup b_{k n}$ and the surface $F_{k} \cap\left(S^{3}-B^{3}\right)$ is connected. We follow [5] in constructing a surgery description of the double branched cover using the surface $F_{k}$. We work with $F_{1}$ and $L_{1}$; the construction for $F_{2}$ and $L_{2}$ is related by a dihedral flype.

Choose a point $v_{i}$ in $D_{i} \cap \partial B^{3}(i=1, \ldots, n)$. Let $G_{k}$ be a spine of $F_{k}$ with the vertex set $\left\{v_{1}, \ldots, v_{n}\right\}$ such that $G_{k} \cap B^{3}$ is a spine of $D_{1} \cup \cdots \cup D_{n} \cup$ $b_{k 1} \cup \cdots \cup b_{k n}$. Let $T_{k} \subset S^{3}-\operatorname{int} B^{3}$ be a spanning tree of $G_{k}$, and $G_{k} / T_{k}$ a spine obtained from $G_{k}$ by contracting $T_{k}$ to a point $v$. We may assume that $N\left(G_{k} / T_{k}\right) \cap F_{k}$ consists of a disk $D_{k 0}$ containing $v$ and mutually disjoint disks $b_{k 1}^{\prime}, \ldots, b_{k m}^{\prime}$ such that $b_{k i}^{\prime} \cap D_{k 0}=\partial b_{k i}^{\prime} \cap \partial D_{k 0}$ are two disjoint arcs in $\partial b_{k i}^{\prime}(i=1, \ldots, m),\left(D_{k 0} \cup b_{k 1}^{\prime} \cup \cdots \cup b_{k m}^{\prime}\right) \cap B_{0}^{3}=\left(b_{k 1} \cup \cdots \cup b_{k n}\right) \cap B_{0}^{3}$, and $\left(D_{10} \cup b_{11}^{\prime} \cup \cdots \cup b_{1 m}^{\prime}\right)-B_{0}^{3}=\left(D_{20} \cup b_{21}^{\prime} \cup \cdots \cup b_{2 m}^{\prime}\right)-B_{0}^{3}$. Let $\varphi: S^{3} \rightarrow S^{3}$ be the double branched cover branched along $\partial D_{k 0}$. Then $M_{L_{k}}^{(2)}$ is obtained from $S^{3}$ by surgery along the framed link $\varphi^{-1}\left(b_{k 1}^{\prime} \cup \cdots \cup b_{k m}^{\prime}\right)$. Note that

$$
\varphi^{-1}\left(\left(b_{k 1}^{\prime} \cup \cdots \cup b_{k m}^{\prime}\right) \cap B_{0}^{3}\right)=\varphi^{-1}\left(\left(b_{k 1} \cup \cdots \cup b_{k n}\right) \cap B_{0}^{3}\right)
$$

are two $n$-rotors and

$$
\varphi^{-1}\left(\left(b_{11}^{\prime} \cup \cdots \cup b_{1 m}^{\prime}\right)-B_{0}^{3}\right)=\varphi^{-1}\left(\left(b_{21}^{\prime} \cup \cdots \cup b_{2 m}^{\prime}\right)-B_{0}^{3}\right) .
$$

Since each $\varphi^{-1}\left(b_{k i}^{\prime}\right)$ is a component of $\varphi^{-1}\left(b_{k 1}^{\prime} \cup \cdots \cup b_{k m}^{\prime}\right)$, it is not hard to see that there is a blackboard framed, oriented link $c_{k 1} \cup \cdots \cup c_{k m}$ such that each $c_{k i}$ corresponds to $b_{k i}^{\prime}$ and both components of $\left(c_{k 1} \cup \cdots \cup c_{k m}\right) \cap \varphi^{-1}\left(B_{0}^{3}\right)$ are oriented $n$-rotors. So $c_{21} \cup \cdots \cup c_{2 m}$ is obtained from $c_{11} \cup \cdots \cup c_{1 m}$ by two oriented $n$-rotations. By Theorem 2.1(iii), the linking matrices of $c_{11} \cup \cdots \cup c_{1 m}$ and $c_{21} \cup \cdots \cup c_{2 m}$ coincide. Since the linking matrix of $c_{k 1} \cup \cdots \cup c_{k m}$ is a relation matrix of the first homology group of $M_{L_{k}}^{(2)}$, we have the conclusion.

Corollary 2.3 and the example in Fig. 1.1 allow us to conclude that not every $n$-rotor has a symmetric disk-band representation with $n$ bands.

Let $F_{L}$ be a Seifert surface of an oriented link $L$. Denote by $\psi: H_{1}\left(F_{L} ; \mathbb{Z}\right)$ $\times H_{1}\left(F_{L} ; \mathbb{Z}\right) \rightarrow \mathbb{Z}$ the Seifert form associated with $F_{L}$ (i.e. $\psi(x, y)=\operatorname{lk}\left(x^{+}, y\right)$, where $x^{+}$denotes the curve $x$ pushed slightly off $F_{L}$ into the positive direction). Choosing an ordered basis for $H_{1}\left(F_{L} ; \mathbb{Z}\right)$ allows us to describe the form $\psi$ by the corresponding Seifert matrix. Let $\mathcal{A}_{L}$ be the Seifert matrix of the form $\psi$ with respect to some ordered basis of $H_{1}\left(F_{L} ; \mathbb{Z}\right)$. 
Let $F_{L}$ be a spanning surface, possibly nonorientable, of an unoriented link $L$. We use the following generalization of Seifert $\left({ }^{2}\right)$ and Goeritz forms defined by Gordon and Litherland in [4]. For the spanning surface $F_{L}$ consider a regular neighborhood, $N\left(F_{L}\right)$, of $F_{L}^{3}$ in $S^{3}-L$. Then $N\left(F_{L}\right)$ is an $I$-bundle over $F_{L}$, and the $\partial I$-bundle $\widetilde{F}_{L}$ is a double cover of $F_{L}$ (possibly disconnected) with the projection map $p: \widetilde{F}_{L} \rightarrow F_{L}$. The bilinear form $\mathcal{G}_{F_{L}}: H_{1}\left(F_{L} ; \mathbb{Z}\right) \times H_{1}\left(F_{L} ; \mathbb{Z}\right) \rightarrow \mathbb{Z}$ defined by $\mathcal{G}_{F_{L}}(x, y)=1 \mathrm{k}\left(p^{-1} x, y\right)$, where $x$ and $y$ are oriented loops in $F_{L}$, is called the Goeritz form associated to the surface $F_{L}$. For an ordered basis of $H_{1}\left(F_{L} ; \mathbb{Z}\right)$ the Goeritz form $\mathcal{G}_{F_{L}}$ is represented by a matrix $G_{F_{L}}$, called the Goeritz matrix of $F_{L}$.

The form $\mathcal{G}_{F_{L}}$ defined over $\mathbb{Z}$ can be extended to a form $\widehat{\mathcal{G}}_{F_{L}}$ over $\mathbb{C}$. We view $\widehat{\mathcal{G}}_{F_{L}}$ as the Hermitian form represented in a basis by the Hermitian matrix $\widehat{G}_{F_{L}}$ (i.e. $\widehat{G}_{F_{L}}=\widehat{\widehat{G}}_{F_{L}}^{t}$ ).

For a spanning surface $F_{L_{k}}$ of $L_{k}=K_{k 1} \cup K_{k 2} \cup \cdots \cup K_{k m}$, the framing of $L_{k}$ is uniquely determined by $F_{L_{k}}$ as follows $\left({ }^{3}\right)$ : Let $K_{k i}^{F_{L_{k}}}$ be a parallel copy of $K_{k i}$ that misses $F_{L_{k}}$. We define the framing $K_{k i}$ to be $\operatorname{lk}\left(K_{k i}, K_{k i}^{F_{L_{k}}}\right)$. We put

$$
e\left(F_{L_{k}}\right)=-\sum_{i} \operatorname{lk}\left(K_{k i}, K_{k i}^{F_{L_{k}}}\right)=-\operatorname{tr}\left(L_{k}\right)
$$

We recall the definition of the Tristram-Levine signature of an oriented link.

Definition $2.4([8,15])$. Let $L$ be an oriented link in $S^{3}$ and let $\omega$ be a complex number with $|\omega|=1, \omega \neq 1$. The Tristram-Levine signature of $L$, denoted by $\sigma_{\omega}(L)$, is the signature of the Hermitian matrix $(1-\omega) \mathcal{A}_{L}+$ $(1-\bar{\omega}) \mathcal{A}_{L}^{t}$, where $\mathcal{A}_{L}$ is the Seifert matrix of $L$.

Definition 2.5 ([4, 10, 11]). Let $L$ be an unoriented link in $S^{3}$, and let $\widehat{L}$ be the link obtained from $L$ by a choice of orientation. The Murasugi signature $\widehat{\sigma}(L)$ of an unoriented link $L$ is defined to be $\widehat{\sigma}(L)=\sigma(\widehat{L})+\operatorname{lk}(\widehat{L})$.

REMARK 2.6. Murasugi showed in [11] that $\sigma(\widehat{L})+\operatorname{lk}(\widehat{L})$ does not depend on the choice of orientation of $L$. So $\widehat{\sigma}(L)$ is an invariant of unoriented links. We shall use later the fact that $\widehat{\sigma}(L)=\operatorname{sign}\left(G_{F_{L}}\right)+\frac{1}{2} e\left(F_{L}\right)$ (see [4]).

3. Unoriented rotation and Murasugi signature. In this section we prove that the Murasugi signature of unoriented links is preserved by any rotation. The result follows from a more general statement (see Theorem

$\left({ }^{2}\right)$ It is a generalization of the symmetrization of the Seifert form.

$\left({ }^{3}\right)$ The regular neighborhood of $K_{k i}$ in $F_{k}$ is the frame knot associated to $K_{k i}$. Its framing, when compared to the standard framing, is given by $l \mathrm{k}\left(K_{k i}, K_{k i}^{F_{L_{k}}}\right)$. 
3.2) that the rotation preserves the characteristic polynomial of the Goeritz matrix (with the special choices of surfaces). In particular Theorem 3.2 allows us to obtain the result mentioned first in [12] that was also proven by Traczyk that the determinant of an unoriented link is preserved by any rotation.

THEOREM 3.1. Let $L_{1}$ and $L_{2}$ be a pair of unoriented n-rotants (with no restrictions on $n)$. Then $\widehat{\sigma}\left(L_{1}\right)=\widehat{\sigma}\left(L_{2}\right)$.

The main result of this section is Theorem 3.2 from which Theorem 3.1 follows.

Let $L_{1}$ and $L_{2}$ be a pair of unoriented rotant links. Consider projections of the links $L_{1}$ and $L_{2}$ onto $\mathbb{R}^{2}$ with rotor parts $R_{1}$ and $R_{2}$ contained in disks $D_{1}$ and $D_{2}$, respectively. We can deform the stator parts $S_{1}$ and $S_{2}$ of the diagrams of $L_{1}$ and $L_{2}$ into the position shown in Figure 3.1.

We color the regions on $\mathbb{R}^{2}$ bounded by the diagrams of $L_{k}$ in a checkerboard manner as in Figure 3.2. Using the black regions we form the spanning surface $F_{L_{k}}$ for $k=1,2$. We choose for a basis of $H_{1}\left(F_{L_{k}} ; \mathbb{Z}\right)$ the anticlockwise oriented boundary curves of the bounded white regions, and we refer to this basis as the standard basis.

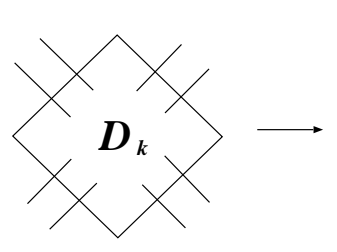

Fig. 3.1

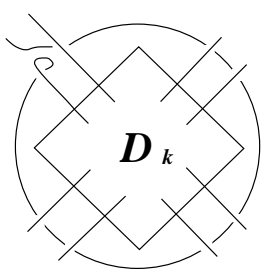$$
\text { (1) }
$$

(1)

We may also assume that the framed links $L_{1}$ and $L_{2}$ obtained from $F_{1}$ and $F_{2}$ respectively form a pair of rotants. By Theorem 2.1, $\operatorname{tr}\left(L_{1}\right)=\operatorname{tr}\left(L_{2}\right)$, so we have $e\left(F_{L_{1}}\right)=e\left(F_{L_{2}}\right)$. This fact, Remark 2.6 and the following theorem imply Theorem 3.1.

With the choices for $F_{k}$ 's and bases of $H_{1}\left(F_{k} ; \mathbb{Z}\right)$ 's, made above, we can formulate the main result of this section.

THEOREM 3.2. Let $G_{F_{k}}(k=1,2)$ be the Goeritz matrices with respect to the standard basis. Then $\operatorname{det}\left(G_{F_{L_{1}}}-\lambda E\right)=\operatorname{det}\left(G_{F_{L_{2}}}-\lambda E\right)$.

Proof. $\left({ }^{4}\right)$ Let $X_{\mathcal{S}_{k}}$ and $X_{\mathcal{R}_{k}}$ be the subsets of the standard basis of $H_{1}\left(F_{L_{k}} ; \mathbb{Z}\right)$ which live entirely in the stator and rotor part respectively, and

$\left({ }^{4}\right)$ We adjust here Traczyk's method [14] to the case of unoriented rotors and Goeritz matrices. 
let $X_{\mathcal{M}_{k}}$ be the complement of $X_{\mathcal{S}_{k}} \cup X_{\mathcal{R}_{k}}$ in the standard basis. Then $X_{\mathcal{M}_{k}}$ is composed of the boundaries of the white regions intersecting the boundary of the rotor. We can have $n$ such regions or just one. We can, however, always assume, modifying the rotor part of the diagram if necessary, that $X_{\mathcal{M}_{k}}$ has $n$ different elements. Consider the submodules $\mathcal{S}_{k}, \mathcal{R}_{k}$ and $\mathcal{M}_{k}$ of $H_{1}\left(F_{L_{k}} ; \mathbb{Z}\right)$ generated by $X_{\mathcal{S}_{k}}, X_{\mathcal{R}_{k}}$ and $X_{\mathcal{M}_{k}}$. We have the following decomposition into the direct sum of $\mathbb{Z}$-modules:

$$
H_{1}\left(F_{L_{k}} ; \mathbb{Z}\right)=\mathcal{S}_{k} \oplus \mathcal{M}_{k} \oplus \mathcal{R}_{k}
$$

Let $v$ denote the generator of $\mathcal{M}_{1}$ intersecting the $y$ axis of the dihedral flype $d$ (Fig. 3.3). There is an action of the cyclic group $\mathbb{Z}_{n}=\left\langle\alpha \mid \alpha^{n}=1\right\rangle$ on $\mathcal{R}_{1} \oplus$ $\mathcal{M}_{1}$ induced by the $2 \pi / n$-rotation about the center of $D_{1}$. Thus the ordered set $X_{\mathcal{M}_{1}}=\left\{v, \alpha(v), \alpha^{2}(v), \ldots, \alpha^{n-1}(v)\right\}$ can be assumed to be a basis of $\mathcal{M}_{1}$. Let $X_{\mathcal{R}_{1}}^{*}$ be a set of generators of $\mathcal{R}_{1}$ formed by choosing one representative from each orbit of the $\mathbb{Z}_{n}$-action on the standard generators of $\mathcal{R}_{1}$ (i.e. $X_{\mathcal{R}_{1}}^{*}=$ $\left.X_{\mathcal{R}_{1}} / \mathbb{Z}_{n}\right)$. We construct a bijection $\eta$ between the sets of standard generators of $H_{1}\left(F_{L_{1}} ; \mathbb{Z}\right)$ and $H_{1}\left(F_{L_{2}} ; \mathbb{Z}\right)$. First, define $\left.\eta\right|_{X_{\mathcal{S}_{1}}}: X_{\mathcal{S}_{1}} \rightarrow X_{\mathcal{S}_{2}}$ to be the identity map since the stator part is unchanged by rotation. The map $\left.\eta\right|_{X_{\mathcal{M}_{1}}}$ : $X_{\mathcal{M}_{1}} \rightarrow X_{\mathcal{M}_{2}}$ is given by $\eta\left(\alpha^{j}(v)\right)=\alpha^{j}(d(v))$ (i.e. $\alpha^{j}(v)$ and $\eta\left(\alpha^{j}(v)\right)$ have the same stator parts). Finally, $\left.\eta\right|_{X_{\mathcal{R}_{1}}}: X_{\mathcal{R}_{1}} \rightarrow X_{\mathcal{R}_{2}}$ is given by $\eta\left(\alpha^{j}(x)\right)=$ $d\left(\alpha^{j}(x)\right)$ for $x \in X_{\mathcal{R}_{1}}$. The bijection $\eta$ extends to an isomorphism $H_{1}\left(F_{L_{1}} ; \mathbb{Z}\right)$ $\rightarrow H_{1}\left(F_{L_{2}} ; \mathbb{Z}\right)$, which is also denoted by $\eta$. We use the isomorphism $\eta$ to identify $H_{1}\left(F_{L_{1}} ; \mathbb{Z}\right)$ with $H_{1}\left(F_{L_{2}} ; \mathbb{Z}\right)$. This identification allows us to drop the indices in $\mathcal{S}_{k}, \mathcal{M}_{k}$ and $\mathcal{R}_{k}$ and write $\mathcal{S}, \mathcal{M}$ and $\mathcal{R}$.
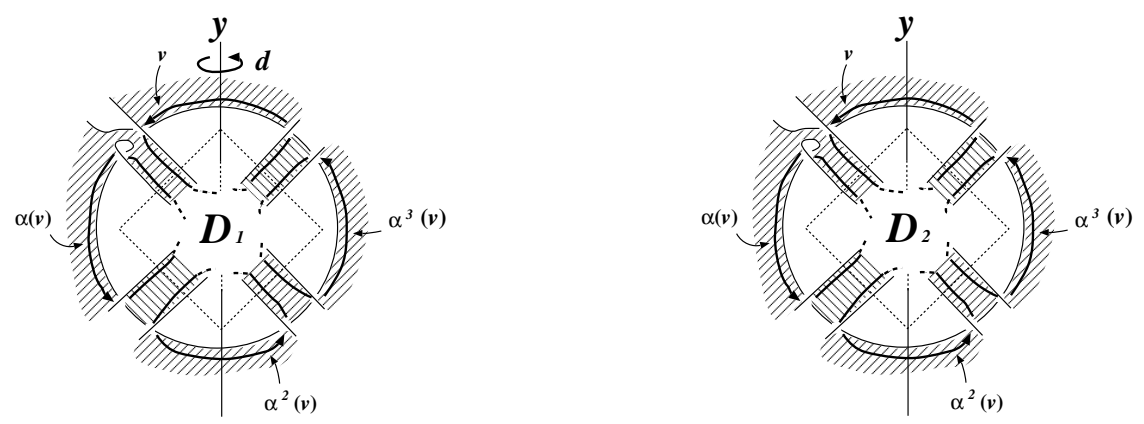

Fig. 3.3

Consider the forms $\mathcal{G}_{1}=\mathcal{G}_{F_{L_{1}}}$ and $\mathcal{G}_{2}=\mathcal{G}_{F_{L_{2}}}$ on the same space $\mathcal{S} \oplus$ $\mathcal{M} \oplus \mathcal{R}$. They have the following properties:

$$
\begin{aligned}
& \mathcal{G}_{2}(x, y)=\mathcal{G}_{1}(x, y) \quad \text { for all } x, y \in \mathcal{S} \oplus \mathcal{M}, \\
& \mathcal{G}_{2}(x, y)=\mathcal{G}_{1}(x, y) \quad \text { for all generators } x, y \in \mathcal{R},
\end{aligned}
$$




$$
\begin{array}{ll}
\mathcal{G}_{1}(x, y)=\mathcal{G}_{1}\left(\alpha^{l}(x), \alpha^{l}(y)\right) & \text { for all generators } x, y \in \mathcal{M} \oplus \mathcal{R}, \\
\mathcal{G}_{2}\left(x, \alpha^{l}(v)\right)=\mathcal{G}_{1}\left(x, \alpha^{-l}(v)\right) & \text { for every generator } x \in \mathcal{R}, \\
\mathcal{G}_{2}\left(\alpha^{l}(x), v\right)=\mathcal{G}_{1}\left(\alpha^{l}(x), v\right) & \text { for every generator } x \in \mathcal{R}, \\
\mathcal{G}_{2}(x, v)=\mathcal{G}_{2}\left(\alpha^{l}(x), \alpha^{-l}(v)\right) & \text { for every generator } x \in \mathcal{R} . \\
\mathcal{G}_{k}(x, y)=0 \text { for all } x \in \mathcal{S}, y \in \mathcal{R}(k=1,2) .
\end{array}
$$

Let $\mathbf{S}, \mathbf{M}$ and $\mathbf{R}$ be the subspaces of $(\mathcal{S} \oplus \mathcal{M} \oplus \mathcal{R}) \otimes \mathbb{C}$ complexifying $\mathcal{S}, \mathcal{M}$ and $\mathcal{R}$, respectively. We have the involution ${ }^{-}: \mathbf{S} \oplus \mathbf{M} \oplus \mathbf{R} \rightarrow \mathbf{S} \oplus \mathbf{M} \oplus \mathbf{R}$ corresponding to the conjugation in the factor $\mathbb{C}$ of the tensor product. The image of $x \in \mathbf{S} \oplus \mathbf{M} \oplus \mathbf{R}$ under this involution is denoted by $\bar{x}$. Using the rotational symmetry of the rotor part we conveniently change the basis of $\mathbf{M}$ and the generating set of $\mathbf{R}$ in the following way. Let $\omega_{j}$ be an $n$th root of unity, $\omega_{j}=e^{2 \pi i j / n}$. We replace the basis $\left\{\alpha^{j}(v) \mid j=0,1, \ldots, n-1\right\}$ of $\mathbf{M}$ by $\left\{\mathbf{v}_{j} \mid \mathbf{v}_{j}=\sum_{l=0}^{n-1} \omega_{j}^{l} \alpha^{l}(v), j=0,1, \ldots, n-1\right\}$. For $\mathbf{R}$ we consider two choices of generating sets that are related by the involution as follows. We replace the set $\left\{\alpha^{j}\left(y_{p}\right) \mid y_{p} \in X_{\mathcal{R}}^{*}, j=0,1, \ldots, n-1\right\}$ by either $\left\{\mathbf{y}_{j, p} \mid \mathbf{y}_{j, p}=\sum_{l=0}^{n-1} \omega_{j}^{l} \alpha^{l}\left(y_{p}\right), y_{p} \in X_{\mathcal{R}}^{*}, j=0,1, \ldots, n-1\right\}$ or $\left\{\overline{\mathbf{y}}_{j, p} \mid \overline{\mathbf{y}}_{j, p}=\sum_{l=0}^{n-1} \bar{\omega}_{j}^{l} \alpha^{l}\left(y_{p}\right), y_{p} \in X_{\mathcal{R}}^{*}, j=0,1, \ldots, n-1\right\}$.

Consider the Hermitian forms $\widehat{\mathcal{G}}_{1}=\widehat{\mathcal{G}}_{F_{L_{1}}}$ and $\widehat{\mathcal{G}}_{2}=\widehat{\mathcal{G}}_{F_{L_{2}}}$, induced by $\mathcal{G}_{1}$ and $\mathcal{G}_{2}$, on the same space $\mathbf{S} \oplus \mathbf{M} \oplus \mathbf{R}$.

These new generating sets for $\mathbf{M} \oplus \mathbf{R}$ satisfy the following conditions:

$$
\begin{aligned}
& \widehat{\mathcal{G}}_{k}\left(\mathbf{v}_{j}, \mathbf{v}_{m}\right)=0 \quad \text { for } j \neq m \text {, where } \mathbf{v}_{j}, \mathbf{v}_{m} \in \mathbf{M} \text { and } k=1,2 \text {, } \\
& \widehat{\mathcal{G}}_{1}\left(\mathbf{x}_{j, p}, \mathbf{v}_{m}\right)=\widehat{\mathcal{G}}_{2}\left(\overline{\mathbf{x}}_{j, p}, \mathbf{v}_{m}\right)=0 \text { for } j \neq m \text {, } \\
& \text { where } \mathbf{x}_{j, p} \in \mathbf{R}_{1}, \overline{\mathbf{x}}_{j, p} \in \mathbf{R}_{2}, \mathbf{v}_{m} \in \mathbf{M} \\
& \widehat{\mathcal{G}}_{1}\left(\mathbf{x}_{j, p}, \mathbf{y}_{m, q}\right)=\widehat{\mathcal{G}}_{2}\left(\overline{\mathbf{x}}_{j, p}, \overline{\mathbf{y}}_{m, q}\right) \text { for } j \neq m \text {, } \\
& \text { where } \mathbf{x}_{j, p}, \mathbf{y}_{m, q} \in \mathbf{R}_{1}, \overline{\mathbf{x}}_{j, p}, \overline{\mathbf{y}}_{m, q} \in \mathbf{R}_{2} \text {, } \\
& \widehat{\mathcal{G}_{1}}\left(\mathbf{x}, \mathbf{y}_{j, p}\right)=\widehat{\mathcal{G}}_{2}\left(\mathbf{x}, \overline{\mathbf{y}}_{j, p}\right)=0 \quad \text { for any } \mathbf{x} \in \mathbf{S}, \mathbf{y}_{j, p} \in \mathbf{R}_{1}, \overline{\mathbf{y}}_{j, p} \in \mathbf{R}_{2} \text {, } \\
& \widehat{\mathcal{G}}_{1}\left(\mathbf{y}_{j, p}, \mathbf{y}_{j, q}\right)=\overline{\widehat{\mathcal{G}}_{2}\left(\overline{\mathbf{y}}_{j, p}, \overline{\mathbf{y}}_{j, q}\right)} \quad \text { for any } \mathbf{y}_{j, p}, \mathbf{y}_{j, q} \in \mathbf{R}_{1}, \overline{\mathbf{y}}_{j, p}, \overline{\mathbf{y}}_{j, q} \in \mathbf{R}_{2} \text {, } \\
& \widehat{\mathcal{G}}_{1}\left(\mathbf{v}_{j}, \mathbf{y}_{j, p}\right)=\overline{\widehat{\mathcal{G}}_{2}\left(\mathbf{v}_{j}, \overline{\mathbf{y}}_{j, p}\right)} \quad \text { for any } \mathbf{v}_{j} \in \mathbf{M}, \mathbf{y}_{j, p} \in \mathbf{R}_{1}, \overline{\mathbf{y}}_{j, p} \in \mathbf{R}_{2} \text {, } \\
& \widehat{\mathcal{G}}_{1}\left(\mathbf{v}_{j}, \mathbf{v}_{j}\right)=\widehat{\mathcal{G}}_{2}\left(\mathbf{v}_{j}, \mathbf{v}_{j}\right) \quad \text { for any } \mathbf{v}_{j} \in \mathbf{M} \text {, } \\
& \widehat{\mathcal{G}}_{1}\left(\mathbf{x}, \mathbf{v}_{j}\right)=\widehat{\mathcal{G}}_{2}\left(\mathbf{x}, \mathbf{v}_{j}\right) \quad \text { for any } \mathbf{x} \in \mathbf{S}, \mathbf{v}_{j} \in \mathbf{M} \text {. }
\end{aligned}
$$

For a given $\omega_{j}, 0 \leq j \leq n-1$, let $W_{j}$ be the subspace of $\mathbf{M} \oplus \mathbf{R}$ defined by choosing its ordered basis in the following way. Take $\mathbf{v}_{j}$ from $\mathbf{M}$ first and $\mathbf{y}_{j, p}$ from $\mathbf{R}$ in any order. To obtain the ordered basis of $\mathbf{M} \oplus \mathbf{R}$ we place the basis of $W_{j}$ before the basis of $W_{j+1}$ for $j=0,1, \ldots, n-1$. Finally, we add the ordered basis of $\mathbf{S}$. We thus obtain an ordered basis of $H_{1}\left(F_{L_{1}} ; \mathbb{C}\right)$. 
Notice that we can construct an ordered basis of $H_{1}\left(F_{L_{2}} ; \mathbb{C}\right)$ by replacing each $\mathbf{y}_{j, p}$ with $\overline{\mathbf{y}}_{j, p}$.

Let $\widehat{G}_{1}$ and $\widehat{G}_{2}$ be the matrices of the forms $\widehat{\mathcal{G}}_{1}$ and $\widehat{\mathcal{G}}_{2}$ respectively in the ordered bases of $\mathbf{S} \oplus \mathbf{M} \oplus \mathbf{R}$ chosen before. Then

$$
\begin{aligned}
& \widehat{G}_{1}=\left(\begin{array}{cccc}
B_{10} & & \mathbf{0} & { }^{t} \bar{S}_{0} \\
& \ddots & & \vdots \\
\mathbf{0} & & B_{1, n-1} & { }^{t} \bar{S}_{n-1} \\
S_{0} & \cdots & S_{n-1} & S
\end{array}\right), \\
& \widehat{G}_{2}=\left(\begin{array}{cccc}
B_{20} & & \mathbf{0} & { }^{t} \bar{S}_{0} \\
& \ddots & & \vdots \\
\mathbf{0} & & B_{2, n-1} & { }^{t} \bar{S}_{n-1} \\
S_{0} & \cdots & S_{n-1} & S
\end{array}\right) .
\end{aligned}
$$

In these bases, $B_{1 j}$ (respectively $B_{2 j}$ ), where $j=0,1, \ldots, n-1$, is the matrix of the restriction of the form $\widehat{\mathcal{G}}_{1}$ (and $\widehat{\mathcal{G}_{2}}$ respectively) to the subspace $W_{j}$ generated by $\left\{\mathbf{v}_{j}\right\} \cup\left\{\mathbf{y}_{j, p} \mid y_{p} \in X_{\mathcal{R}_{1}}^{*}\right\}\left(\left\{\mathbf{v}_{j}\right\} \cup\left\{\overline{\mathbf{y}}_{j, p} \mid y_{p} \in X_{\mathcal{R}_{1}}^{*}\right\}\right.$ respectively). Finally, the restrictions of $\widehat{\mathcal{G}_{1}}$ and $\widehat{\mathcal{G}}_{2}$ to the stator part are the same for $\widehat{\mathcal{G}_{1}}$ and $\widehat{\mathcal{G}_{2}}$ and denoted by $S$. Notice that $B_{1 k}^{t}=B_{2 k}, S_{l}=\left(\mathbf{s}_{l 1} \mathbf{0} \cdots \mathbf{0}\right)$, and $\mathbf{s}_{l 1}$ is the first column of $S_{l}$.

The matrices $M_{k}=\widehat{G}_{k}-\lambda E(k=1,2)$ satisfy the conditions of Proposition 2.9 of Traczyk [14] for any real number $\lambda$. Thus $\operatorname{det}\left(M_{1}\right)=\operatorname{det}\left(M_{2}\right)$ for any real $\lambda$. So the determinants are equal for any complex $\lambda$ as well.

4. Oriented rotation and Tristram-Levine signature. In this section we extend the method developed by Traczyk in [14] in order to show that orientation-preserving rotations (see Fig. 2.2(a)) preserve the Conway polynomial. We show that the characteristic polynomial of the Hermitian form associated with the Seifert form of an appropriately chosen Seifert surface is invariant under orientation-preserving rotations. In particular we prove the following result.

THEOREM 4.1. Let $L_{1}$ and $L_{2}$ be a pair of orientation-preserving $n$ rotants. Then $\sigma_{\omega}\left(L_{1}\right)=\sigma_{\omega}\left(L_{2}\right)$.

The main result of this section is Theorem 4.2 from which Theorem 4.1 follows.

Let $S^{2}$ be the sphere of a projection of a link $L$, and $F_{L}$ the Seifert surface of $L$ obtained from the diagram of $L$ by the Seifert algorithm. Let $H$ be a trivalent graph that consists of the Seifert circles and the cores of the bands. Let $R_{1}, \ldots, R_{m}$ be the components of $S^{2}-H$ which are not bounded 
by Seifert circles. Assign the anti-clockwise orientation to each boundary curve of the regions $R_{i}(i=1, \ldots, m)$; then these curves are generators of $H_{1}\left(F_{L} ; \mathbb{Z}\right)$. Whenever we refer to generators of $H_{1}\left(F_{L} ; \mathbb{Z}\right)$, we mean this particular set of standard generators for the Seifert surface $F_{L}$.

Let $L_{1}$ and $L_{2}$ be a pair of orientation-preserving $n$-rotant diagrams.

We deform the diagrams $L_{k}(k=1,2)$ on $S^{2}$ into the position for which our computation is feasible, as in [14]. Let $D_{k}$ be a disk in $S^{2}$ such that $D_{k}^{r}=D_{k} \cap L_{k}$ is the rotor part of the diagram $L_{k}(k=1,2)$, and $D_{k}^{s}=\bar{D}_{k} \cap L_{k}$ the stator part $\left(\bar{D}_{k}=S^{2}-\operatorname{int} D_{k}\right)$. The rotors and stators constructed above are all $n$-tangles. We deform the stator part $D_{1}^{s}=D_{2}^{s}$ to the form shown in Fig. 4.1. By doing so we obtain an outermost Seifert circle $C$ in $\bar{D}$ that is parallel to $\partial D_{k}$. Let $\bar{D}_{C}$ be the region which is bounded by $C$ and $\partial D_{k}$ in $\bar{D}$. We extend the rotational symmetries of the rotor parts $D_{k}^{r}(k=1,2)$ to the parts embedded in $D_{k} \cup \bar{D}_{C}$, i.e., we may assume that $D_{k} \cup \bar{D}_{C}(k=1,2)$ contain $n$-rotors.
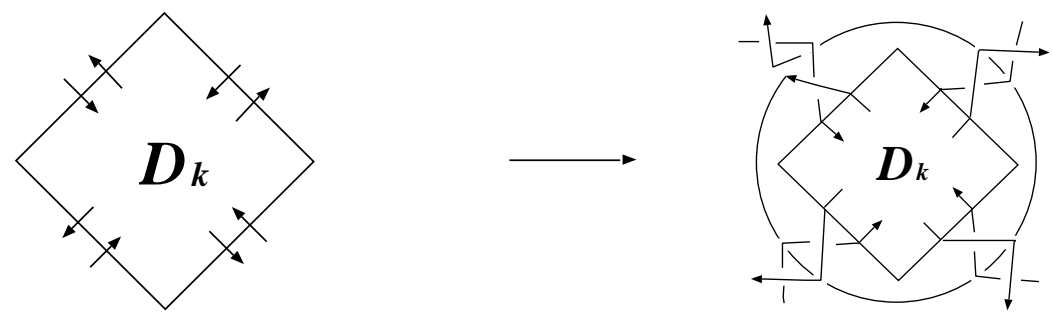

Fig. 4.1

Let $F_{L_{k}}(k=1,2)$ be the Seifert surface for $L_{k}$ (Fig. 4.2), and let $\mathcal{A}_{L_{k}}$ be the corresponding Seifert matrix of $L_{k}, k=1,2$.

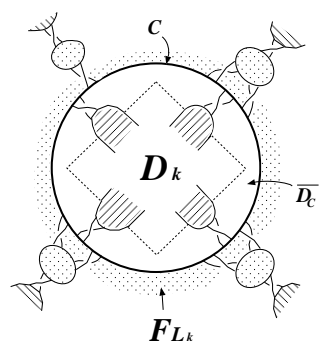

Fig. 4.1

Let $\xi$ be a complex number and let $X_{L_{k}}=\xi \mathcal{A}_{L_{k}}+\bar{\xi} \mathcal{A}_{L_{k}}^{t}$ be the Hermitian matrix that represents the Hermitian form $\theta(x, y)=\xi \psi(x, y)+\bar{\xi} \psi(y, x)$, $x, y \in H_{1}\left(F_{L_{k}} ; \mathbb{Z}\right)$. 
With the choices for the Seifert surfaces $F_{k}$ and the bases of $H_{1}\left(F_{k}\right)$ made above, we can formulate the main result of this section.

THEOREM 4.2. The characteristic polynomials of the Hermitian matrices $X_{L_{1}}$ and $X_{L_{2}}$ coincide.

Proof. We consider three submodules $\mathcal{S}_{k}, \mathcal{R}_{k}$ and $\mathcal{M}_{k}$ of $H_{1}\left(F_{L_{k}} ; \mathbb{Z}\right)$, where $\mathcal{S}_{k}, \mathcal{R}_{k}$ and $\mathcal{M}_{k}$ are generated by the sets $X_{S_{k}}, X_{R_{k}}$, and $X_{M_{k}}$ of the standard generators of $H_{1}\left(F_{L_{k}} ; \mathbb{Z}\right)$ which live entirely in the stator part $\bar{D}$, rotor part $D_{k}$, and partially in $\bar{D}$ and $D_{k}(k=1,2)$, respectively. We have the following decomposition of the module $H_{1}\left(F_{L_{k}} ; \mathbb{Z}\right)$ into the direct sum of its submodules:

$$
H_{1}\left(F_{L_{k}} ; \mathbb{Z}\right)=\mathcal{S}_{k} \oplus\left(\mathcal{M}_{k}+\mathcal{R}_{k}\right) \quad(k=1,2) .
$$

Let $v$ denote the generator of $\mathcal{M}_{1}$ intersecting the $y$ axis of the dihedral flype $d$ (Fig. 4.3). There is an action of the cyclic group $\mathbb{Z}_{n}=\left\langle\alpha \mid \alpha^{n}=1\right\rangle$ on $\mathcal{M}_{1}+\mathcal{R}_{1}$ induced by the $2 \pi / n$-rotation about the center of $D_{1}$. The set $X_{M_{1}}=\left\{v, \alpha(v), \alpha^{2}(v), \cdots, \alpha^{n-1}(v)\right\}$ is a generating set of $\mathcal{M}_{1}$ (not necessarily a basis). We also identify $\alpha^{j}(v)$ with the generator of $\mathcal{M}_{2}$ that coincides with the $\alpha^{j}(v)$ of $\mathcal{M}_{1}$ in $\bar{D}_{C}$. The submodule $\mathcal{R}_{1}$ is generated by the set $\left\{\alpha^{j}(x) \mid x \in X_{R_{1}}, j=0,1, \ldots, n-1\right\}$. Since $D_{2}$ is the image of $D_{1}$ under the dihedral flype $d$ about the $y$ axis which crosses $v, \mathcal{R}_{2}$ is generated by $\left\{d\left(\alpha^{j}(x)\right) \mid x \in X_{R_{1}}, j=0,1, \ldots, n-1\right\}$ (Fig. 4.3). In order to compare $\psi_{1}$ with $\psi_{2}$, we identify the generator $\alpha^{j}(x)$ of $\mathcal{R}_{1}$ with the generator $d\left(\alpha^{j}(x)\right) \in \mathcal{R}_{2}(j=0,1, \ldots, n-1)$.
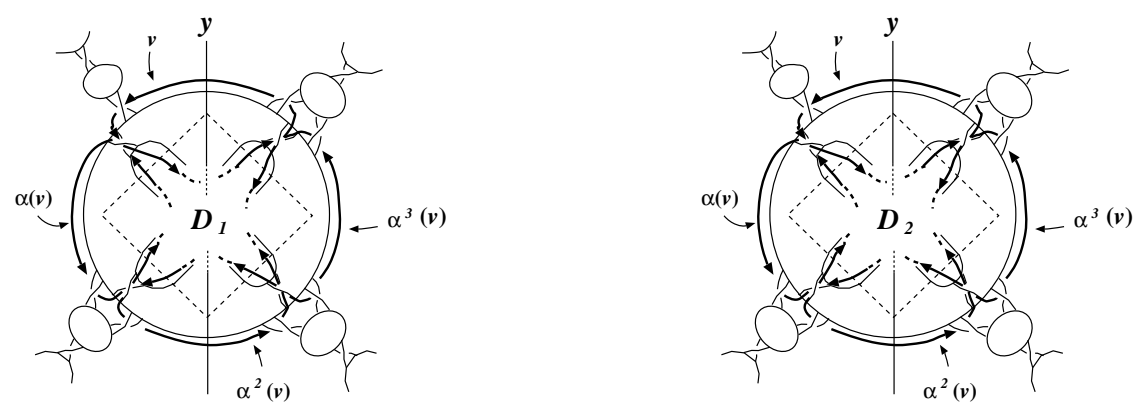

Fig. 4.3

Using these identifications we can consider both forms $\psi_{1}$ and $\psi_{2}$ on the same submodules $\mathcal{S}, \mathcal{M}$ and $\mathcal{R}$ (indices are no more needed) and derive the following relationships between them:

$$
\begin{array}{ll}
\psi_{2}(x, y)=\psi_{1}(x, y) & \text { for all } x, y \in \mathcal{S}+\mathcal{M}, \\
\psi_{2}(x, y)=\psi_{1}(y, x) & \text { for all } x, y \in \mathcal{R},
\end{array}
$$




$$
\begin{aligned}
& \psi_{2}\left(x, \alpha^{j}(v)\right)=\psi_{1}\left(\alpha^{-j}(v), x\right), \\
& \psi_{2}\left(\alpha^{j}(v), x\right)=\psi_{1}\left(x, \alpha^{-j}(v)\right) \quad \text { for all } x \in \mathcal{R}(j=0,1, \ldots, n-1), \\
& \psi_{1}(x, y)=\psi_{1}(y, x)=0=\psi_{2}(x, y)=\psi_{2}(y, x) \quad \text { for all } x \in \mathcal{S}, y \in \mathcal{R} .
\end{aligned}
$$

Using these relations, we obtain the corresponding relations between $\theta_{1}$ and $\theta_{2}$. Let $\mathbf{S}, \mathbf{M}$ and $\mathbf{R}$ be the complexifications of the subspaces $\mathcal{S}, \mathcal{M}$ and $\mathcal{R}$ of $\mathcal{S} \oplus(\mathcal{M}+\mathcal{R}) \otimes \mathbb{C}$ respectively. There is a well defined involution - : $\mathbf{S} \oplus(\mathbf{M}+\mathbf{R}) \rightarrow \mathbf{S} \oplus(\mathbf{M}+\mathbf{R})$ corresponding to the conjugation in the factor $\mathbb{C}$ of the tensor product. We denote by $\bar{x}$ the image of $x \in \mathbf{S} \oplus(\mathbf{M}+\mathbf{R})$ under this involution. The following identities follow from those given above:

$$
\begin{array}{ll}
\theta_{2}(x, y)=\theta_{1}(x, y) & \text { for all } x, y \in \mathbf{S} \oplus \mathbf{M}, \\
\theta_{2}(x, y)=\theta_{1}(y, x)=\overline{\theta_{1}(x, y)} & \text { for all generators } x, y \in \mathbf{R}, \\
\theta_{2}(x, y)=\overline{\theta_{1}(\bar{x}, \bar{y})} & \text { for all } x, y \in \mathbf{R}, \\
\theta_{1}(x, y)=\theta_{1}\left(\alpha^{j}(x), \alpha^{j}(y)\right) & \text { for all generator } x, y \in \mathbf{M}+\mathbf{R}, \\
\theta_{2}\left(x, \alpha^{j}(v)\right)=\theta_{1}\left(\alpha^{-j}(v), x\right) & \text { for every generator } x \text { of } \mathbf{R}, \\
\theta_{2}\left(\alpha^{j}(x), v\right)=\overline{\theta_{1}\left(\alpha^{j}(x), v\right)} & \text { for every generator } x \text { of } \mathbf{R}, \\
\theta_{2}(x, v)=\theta_{2}\left(\alpha^{j}(x), \alpha^{-j}(v)\right) & \text { for every generator } x \in \mathbf{R}, \\
\theta_{k}(x, y)=0 & \text { for all } x \in \mathbf{S}, y \in \mathbf{R}, k=1,2 .
\end{array}
$$

In order to define the Hermitian matrices $H_{L_{k}}$ representing $\theta_{k}(k=1,2)$, we first choose a basis of $H_{1}\left(F_{L_{k}} ; \mathbb{C}\right)$ that is formed using the generators of $H_{1}\left(F_{L_{k}} ; \mathbb{Z}\right)$ in the following way. Set again $\omega_{j}=e^{2 \pi i j / n}(j=1, \ldots, n)$. We replace the generating set $\left\{\alpha^{j}(v) \mid j=0,1, \ldots, n-1\right\}$ of $\mathbf{M}$ by $\left\{\mathbf{v}_{j} \mid \mathbf{v}_{j}=\sum_{l=0}^{n-1} \omega_{j}^{l} \alpha^{l}(v), j=0,1, \ldots, n-1\right\}$. For $\mathbf{R}$ we consider two choices of generating sets related by the involution - We replace $\left\{\alpha^{j}\left(y_{p}\right) \mid\right.$ $\left.y_{p} \in X_{\mathcal{R}}, j=0,1, \ldots, n-1\right\}$ by either $\left\{\mathbf{y}_{j, p} \mid \mathbf{y}_{j, p}=\sum_{l=0}^{n-1} \omega_{j}^{l} \alpha^{l}\left(y_{p}\right)\right.$, $\left.y_{p} \in X_{\mathcal{R}}, j=0,1, \ldots, n-1\right\}$ or $\left\{\overline{\mathbf{y}}_{j, p} \mid \overline{\mathbf{y}}_{j, p}=\sum_{l=0}^{n-1} \bar{\omega}_{j}^{l} \alpha^{l}\left(y_{p}\right), y_{p} \in X_{\mathcal{R}}\right.$, $j=0,1, \ldots, n-1\}$.

We thus obtain a new generating set for $\mathbf{M}_{k}+\mathbf{R}_{k}$. The following relationships hold:

$$
\begin{array}{ll}
\theta_{k}\left(\mathbf{v}_{j}, \mathbf{v}_{m}\right)=0 & \text { for } j \neq m, \text { where } \mathbf{v}_{j}, \mathbf{v}_{m} \in \mathbf{M}, k=1,2, \\
\theta_{1}\left(\mathbf{x}_{j, p}, \mathbf{v}_{m}\right)=\theta_{2}\left(\overline{\mathbf{x}}_{j, p}, \mathbf{v}_{m}\right)=0 & \text { for } j \neq m, \\
& \text { where } \mathbf{x}_{j, p} \in \mathbf{R}_{1}, \overline{\mathbf{x}}_{j, p} \in \mathbf{R}_{2}, \mathbf{v}_{m} \in \mathbf{M}, \\
\theta_{1}\left(\mathbf{x}_{j, p}, \mathbf{y}_{m, q}\right)=\theta_{2}\left(\overline{\mathbf{x}}_{j, p}, \overline{\mathbf{y}}_{m, q}\right) & \text { for } j \neq m, \\
& \text { where } \mathbf{x}_{j, p}, \mathbf{y}_{m, q} \in \mathbf{R}_{1}, \overline{\mathbf{x}}_{j, p}, \overline{\mathbf{y}}_{m, q} \in \mathbf{R}_{2}, \\
\theta_{1}\left(\mathbf{x}, \mathbf{y}_{j, p}\right)=\theta_{2}\left(\mathbf{x}, \overline{\mathbf{y}}_{j, p}\right)=0 & \text { for any } \mathbf{x} \in \mathbf{S}, \mathbf{y}_{j, p} \in \mathbf{R}_{1}, \overline{\mathbf{y}}_{j, p} \in \mathbf{R}_{2}, \\
\theta_{1}\left(\mathbf{y}_{j, p}, \mathbf{y}_{j, q}\right)=\bar{\theta}_{2}\left(\overline{\mathbf{y}}_{j, p}, \overline{\mathbf{y}}_{j, q}\right) & \text { for any } \mathbf{y}_{j, p}, \mathbf{y}_{j, q} \in \mathbf{R}_{1}, \overline{\mathbf{y}}_{j, p}, \overline{\mathbf{y}}_{j, q} \in \mathbf{R}_{2},
\end{array}
$$




$$
\begin{array}{ll}
\theta_{1}\left(\mathbf{v}_{j}, \mathbf{y}_{j, p}\right)=\overline{\theta_{2}\left(\mathbf{v}_{j}, \overline{\mathbf{y}}_{j, p}\right)} & \text { for any } \mathbf{v}_{j} \in \mathbf{M}, \mathbf{y}_{j, p} \in \mathbf{R}_{1}, \overline{\mathbf{y}}_{j, p} \in \mathbf{R}_{2}, \\
\theta_{1}\left(\mathbf{v}_{j}, \mathbf{v}_{j}\right)=\theta_{2}\left(\mathbf{v}_{j}, \mathbf{v}_{j}\right) & \text { for any } \mathbf{v}_{j} \in \mathbf{M}, \\
\theta_{1}\left(\mathbf{x}, \mathbf{v}_{j}\right)=\theta_{2}\left(\mathbf{x}, \mathbf{v}_{j}\right) & \text { for any } \mathbf{x} \in \mathbf{S}, \mathbf{v}_{j} \in \mathbf{M} .
\end{array}
$$

Take the subspace $W_{j}$ of $\mathbf{M} \oplus \mathbf{R}$ corresponding to $\omega_{j}$, and choose its ordered basis by taking $\mathbf{v}_{j}$ from $\mathbf{M}$ first $\left({ }^{5}\right)$ and the rest of a basis of $W_{j}$ from the generating set $\mathbf{y}_{j, p}$ of $\mathbf{R}$ in any order. To obtain the ordered basis of $\mathbf{M} \oplus \mathbf{R}$ we place the basis of $W_{j}$ before the basis of $W_{j+1}$ for $j=0,1, \ldots, n-1$. Finally, we add the ordered basis of $\mathbf{S}$. Then we have an ordered basis of $H_{1}\left(F_{L} ; \mathbb{C}\right)$. We also obtain an ordered basis of $H_{1}\left(F_{L_{2}} ; \mathbb{C}\right)$ by replacing each $\mathbf{y}_{j, p}$ with $\overline{\mathbf{y}}_{j, p}$.

We now obtain the matrices of the forms $\theta_{1}$ and $\theta_{2}$ in the ordered basis of $\mathbf{S} \oplus(\mathbf{M}+\mathbf{R})$ :

$$
\begin{aligned}
H_{L_{1}}^{\prime}= & \left(\begin{array}{cccc}
B_{10} & & \mathbf{0} & { }^{t} \bar{S}_{0} \\
& \ddots & & \vdots \\
\mathbf{0} & & B_{1, n-1} & { }^{t} \bar{S}_{n-1} \\
S_{0} & \cdots & S_{n-1} & S
\end{array}\right), \\
H_{L_{2}}^{\prime}= & \left(\begin{array}{cccc}
B_{20} & & \mathbf{0} & t \bar{S}_{0} \\
& \ddots & & \vdots \\
\mathbf{0} & & B_{2, n-1} & t \bar{S}_{n-1} \\
S_{0} & \cdots & S_{n-1} & S
\end{array}\right) .
\end{aligned}
$$

In those bases, $B_{1 j}$ (respectively $B_{2 j}$ ), where $j=0,1, \ldots, n-1$, is the matrix of the restriction of the form $\theta_{1}$ (and $\theta_{2}$ respectively) to the subspace $W_{j}$ generated by $\left\{\mathbf{v}_{j}\right\} \cup\left\{\mathbf{y}_{j, p} \mid y_{p} \in X_{\mathcal{R}_{1}}\right\}\left(\left\{\mathbf{v}_{j}\right\} \cup\left\{\overline{\mathbf{y}}_{j, p} \mid y_{p} \in X_{\mathcal{R}_{1}}\right\}\right.$ respectively). Finally, the restriction to the stator part, $S$, is the same for both $\theta_{1}$ and $\theta_{2}$. Notice that $B_{1 k}^{t}=B_{2 k}, S_{l}=\left(\mathbf{s}_{l 1} \mathbf{0} \cdots \mathbf{0}\right)$, and $\mathbf{s}_{l 1}$ is the first column of $S_{l}$.

The matrices $M_{k}=H_{L_{k}}^{\prime}-\lambda E(k=1,2)$ satisfy the conditions of Proposition 2.9 of [14] for any real number $\lambda\left({ }^{6}\right)$. Thus $\operatorname{det}\left(M_{1}\right)=\operatorname{det}\left(M_{2}\right)$ for any real $\lambda$. So the determinants are equal for any complex number $\lambda$ as well.

5. Counterexamples. It was proven in [1] that any pair of oriented 3or 4-rotant links share the same Homflypt polynomial (in particular, Conway polynomial). In [14] Traczyk showed that a pair of orientation-preserving $n$-rotant links share the same Conway polynomial. On the other hand, for

$\left({ }^{5}\right)$ If $\mathbf{v}_{j}=0$, which can happen if the generating set $\left\{v, \alpha(v), \alpha^{2}(v), \cdots, \alpha^{n-1}(v)\right\}$ is not a basis of $\mathbf{M}$, we skip this element when building a basis of $H_{1}\left(F_{L} ; \mathbb{C}\right)$.

$\left({ }^{6}\right)$ We can use Proposition 2.9 even if some vectors $w_{j} \in W_{i, j}$ may be 0 . In such a case the block $W_{i, j}$ is orthogonal to the other factors $\left(S\right.$ and $W_{i, j^{\prime}}, j^{\prime} \neq j$ ). 
orientation-reversing $n$-rotants $(n \geq 6)$, the invariance was an open question. We present an example of a pair of 6-rotant knots with different Conway polynomials and different Jones polynomials. Therefore, the invariance in [1] of the Conway polynomial and the Jones polynomial for the orientationreversing rotant links is the best possible. We should also stress that the rotants described in Fig. 5.1 have different Jones and Conway polynomials, but they share the same determinant and the same homology of the corresponding double branched covers.

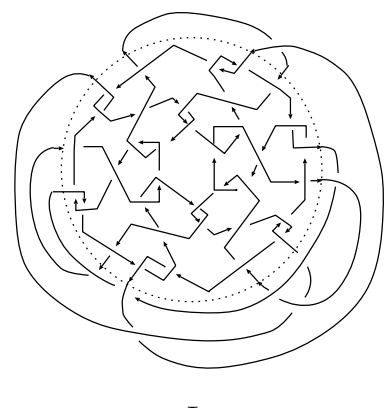

$L_{1}$

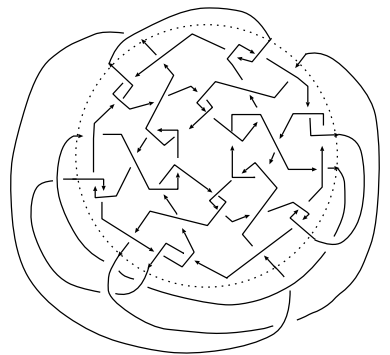

$L_{2}$

Fig. 5.1

Let $L_{1}$ and $L_{2}$ be the knots (6-rotants) illustrated in Fig. 5.1. Using the program KNOT [7], we find the following.

The Conway polynomials (with the skein relation $\nabla_{L_{+}}-\nabla_{L_{-}}=z \nabla_{L_{0}}$ ) are different:

$$
\begin{aligned}
\nabla_{L_{1}}(z)= & 1+3 z^{2}-37 z^{4}+17 z^{6}-3 z^{8}-2 z^{10}-59 z^{12} \\
& -34 z^{14}-55 z^{16}-48 z^{18}-10 z^{20}-4 z^{22}-z^{24} \\
\nabla_{L_{2}}(z)= & 1+3 z^{2}-25 z^{4}-116 z^{8}-57 z^{10}-174 z^{12}-157 z^{14} \\
& -119 z^{16}-102 z^{18}-37 z^{20}-8 z^{22}-z^{24}
\end{aligned}
$$

The Jones polynomials (with the skein relation $t^{-1} V_{L_{+}}-t V_{L_{-}}=(\sqrt{t}-$ $\left.1 / \sqrt{t}) V_{L_{0}}\right)$ are different:

$$
\begin{aligned}
V_{L_{1}}= & t^{23}-16 t^{22}+131 t^{21}-713 t^{20}+2881 t^{19}-9193 t^{18}+24058 t^{17} \\
& -52926 t^{16}+99534 t^{15}-161854 t^{14}+229195 t^{13}-283357 t^{12} \\
& +304679 t^{11}-280476 t^{10}+211413 t^{9}-112418 t^{8}+7697 t^{7}+77824 t^{6} \\
& -127092 t^{5}+136195 t^{4}-114114 t^{3}+77214 t^{2}-41391 t+16087 \\
& -2934 t^{-1}-1501 t^{-2}+1760 t^{-3}-954 t^{-4}+343 t^{-5}-84 t^{-6}+13 t^{-7}-t^{-8},
\end{aligned}
$$




$$
\begin{aligned}
V_{L_{2}}= & t^{23}-16 t^{22}+131 t^{21}-713 t^{20}+2881 t^{19}-9193 t^{18}+24057 t^{17} \\
& -52919 t^{16}+99503 t^{15}-161752 t^{14}+228932 t^{13}-282808 t^{12} \\
& +303730 t^{11}-279098 t^{10}+209727 t^{9}-110701 t^{8}+6314 t^{7}+78540 t^{6} \\
& -126958 t^{5}+135242 t^{4}-112578 t^{3}+75451 t^{2}-39756 t+14823 \\
& -2118 t^{-1}-1933 t^{-2}+1941 t^{-3}-1010 t^{-4}+354 t^{-5}-85 t^{-6}+13 t^{-7}-t^{-8} .
\end{aligned}
$$

Their homology groups are the same: $H_{1}\left(M_{L_{1}}^{2} ; \mathbb{Z}\right)=H_{1}\left(M_{L_{2}}^{2} ; \mathbb{Z}\right)=$ $\mathbb{Z} / 3 \oplus \mathbb{Z} / 397449$. Their determinants coincide as well: $\Delta_{L_{1}}(-1)=\Delta_{L_{2}}(-1)=$ -1192347 (here $\Delta_{L}(t)=\nabla_{L}(z)$ for $z=\sqrt{t}-1 / \sqrt{t}$ ).

\section{References}

[1] R. P. Anstee, J. H. Przytycki and D. Rolfsen, Knot polynomials and generalized mutation, Topology Appl. 32 (1989), 237-249; http://xxx.lanl.gov/abs/math.GT/ 0405382.

[2] R. I. Brooks, C. A. B. Smith, A. H. Stone and W. Tutte, The dissection of rectangles into squares, Duke Math. J. 7 (1940), 312-340.

[3] J. Dymara, T. Januszkiewicz and J. H. Przytycki, Symplectic structure on colorings, Lagrangian tangles and Tits buildings, preprint, 2001.

[4] C. McA. Gordon and R. A. Litherland, On the signature of a link, Invent. Math. 47 (1978), 53-69.

[5] M. Ishiwata, J. H. Przytycki and A. Yasuhara, Branched covers of tangles in threeballs, Canad. Math. Bull. 46 (2003), 356-364; http://arxiv.org/pdf/math.GT/ 0109046.

[6] G. T. Jin and D. Rolfsen, Some remarks on rotors in link theory, Can. Math. Bull. 34 (1991), 480-484.

[7] K. Kodama, KNOT (Software), http://www.math.kobe-u.ac.jp/ kodama/e-index. html.

[8] J. Levine, Polynomial invariants of knots of codimension 2, Ann. of Math. 84 (1966), $537-544$.

[9] J. M. Montesinos, Surgery on links and double branched covers of $S^{3}$, in: Knots, Groups and 3-Manifolds, Ann. of Math. Stud. 84, Princeton Univ. Press, 1975, 227-259.

[10] K. Murasugi, On a certain numerical invariant of link types, Trans. Amer. Math. Soc. 117 (1965), 387-422.

[11] -, On the signature of links, Topology 9 (1970), 283-298.

[12] J. H. Przytycki, Search for different links with the same Jones' type polynomials: Ideas from graph theory and statistical mechanics, in: Panoramas of Mathematics, Banach Center Publ. 34, Inst. Math., Polish Acad. Sci., 1995, 387-422.; http:// xxx.lanl.gov/abs/math.GT/0405447.

[13] —, Skein module deformations of elementary moves on links, Geom. Topol. Monogr. 4 (2002), 313-335; http://xxx.lanl.gov/abs/math.GT/0312527.

[14] P. Traczyk, Conway polynomial and oriented rotant links, Geom. Dedicata, to appear.

[15] A. G. Tristram, Some cobordism invariants for links, Proc. Cambridge Philos. Soc. 66 (1969), 251-264. 
[16] H. F. Trotter, Homology of group systems with applications to knot theory, Ann. of Math. 76 (1962), 464-498.

[17] W. T. Tutte, Codichromatic graphs, J. Combin. Theory Ser. B 16 (1974), 168-174. [18] -, Rotors in graph theory, Ann. Discrete Math. 6 (1980), 343-347.

M. K. Dąbkowski

Department of Mathematics

University of Texas at Dallas

Richardson, TX 75083-0688, U.S.A.

E-mail: mdab@utdallas.edu

J. H. Przytycki

Department of Mathematics

The George Washington University

Washington, DC 20052, U.S.A.

E-mail: przytyck@gwu.edu
M. Ishiwata

Department of Mathematics Tokyo Woman's Christian University Zempukuji 2-6-1, Suginamiku Tokyo 167-8585, Japan E-mail: mako@lab.twcu.ac.jp

A. Yasuhara Department of Mathematics Tokyo Gakugei University Nukuikita 4-1-1, Koganei Tokyo 184-8501, Japan

E-mail: yasuhara@u-gakugei.ac.jp

Received 12 May 2004; in revised form 25 November 2004 